\title{
Auf zu neuen Ufern
}

\section{Off to Pastures New}

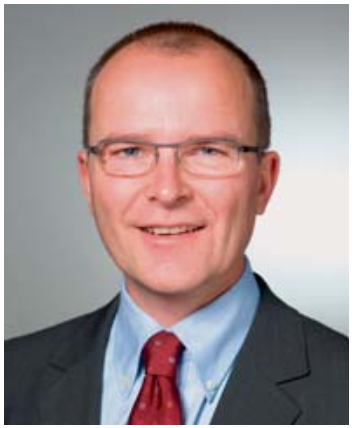

Prof. Dr. med. Bernward Passlick
Zweiundzwanzig Jahre nach ihrer Gründung ist die Deutsche Gesellschaft für Thoraxchirurgie (DGT) mit Beginn dieses Jahres eine Kooperation mit dem Thieme Verlag eingegangen und hat das Zentralblatt für Chirurgie zu ihrem offiziellen Publikationsorgan gewählt. Damit hat die Gesellschaft einen wichtigen Schritt hin zu einem vollständigen Angebot einer wissenschaftlichen Gesellschaft getan. Wichtige Anforderungen der Gesellschaft (offizielles Publikationsorgan, Deutschsprachigkeit, Vorhandensein eines signifikanten Impact Factors) wurden vom Zentralblatt für Chirurgie gleichermaßen erfüllt. Insbesondere ist es dem Thieme Verlag gelungen, ein Angebot zu unterbreiten, welches sich mit den Vorstellungen und Anforderungen einer mittelgroßen wissenschaftlichen Gesellschaft deckt.

Es ist vorgesehen, neben zwei regulären Ausgaben des Zentralblatt für Chirurgie im Februar und Juni, die einen thoraxchirurgischen Schwerpunkt haben werden, jedes Jahr ein Sonderheft „Thoraxchirurgie“ im September/Oktober zum Jahreskongress der DGT herauszugeben. Dieses wird zusätzlich zu den Originalarbeiten auch die Kongressabstracts in zitierfähiger Form beinhalten. Darüber hinaus werden in den thoraxchirurgisch geprägten Ausgaben des Zentralblatts CMEzertifizierte Übersichtsarbeiten zu thoraxchirur- gischen Themen publiziert werden, sowie die international veröffentlichten thoraxchirurgischen Arbeiten in Form von Kurzreferaten vorgestellt. Der Vorstand der DGT dankt dem Team des Thieme Verlags für die hervorragende und intensive Begleitung dieses Projekts, das mit dem vorliegenden Heft 1 seinen erfolgreichen Start findet. Als thoraxchirurgischer Herausgeber wird Herr PD Dr. Paul Schneider, Berlin, fungieren, der die thoraxchirurgischen Arbeiten koordiniert. Der Vorstand der DGT ruft seine Mitglieder auf, das neue Publikationsorgan intensiv zu nutzen, insbesondere da das Zentralblatt nicht nur Originalarbeiten, sondern auch Übersichtsarbeiten, Reviews und sogar Videofilme in zitierfähiger Form publizieren kann.

Das vorliegende Heft beschäftigt sich mit einem originär thoraxchirurgischen Thema, der Trachealchirurgie. Dabei werden neben historischen Aspekten auch die tracheobronchialen Verletzungen beschrieben. Wir wünschen uns, dass sich die Kooperation zwischen dem Zentralblatt für Chirurgie und der DGT zu einem lebendigen Austausch entwickelt.

Prof. Dr. med. Bernward Passlick Präsident der Deutschen Gesellschaft für Thoraxchirurgie 